\title{
Carbon-ion radiotherapy subsequent to balloon-occluded retrograde transvenous obliteration for hepatocellular carcinoma with hepatic encephalopathy: a multidisciplinary approach
}

\author{
Naoto Osu ${ }^{1}$ (1) Shintaro Shiba ${ }^{1,2} \cdot$ Kei Shibuya $^{1,2} \cdot$ Shohei Okazaki ${ }^{2} \cdot$ Yuhei Miyasaka $^{2} \cdot$ Masahiko Okamoto $^{1} \cdot$ \\ Tatsuya Ohno'
}

Received: 19 January 2021 / Accepted: 19 March 2021 / Published online: 3 April 2021

(c) The Author(s) 2021

\begin{abstract}
Radical treatments of hepatocellular carcinoma (HCC) with hepatic encephalopathy (HE) can be often difficult due to poor liver function or disturbance of consciousness. An effective treatment requires a combinatorial approach incorporating a treatment for HE and radical therapy for HCC that does not compromise liver function. Here, we report a case of a 78-yearold Japanese male with HCC and HE caused by splenorenal shunt. Serum ammonia levels were high. He was not suitable for surgery, percutaneous radiofrequency ablation, or transarterial chemoembolization due to the location of the tumor and poor liver function, which included HE. Thus, he underwent BRTO, with an immediate improvement in both HE and serum ammonia levels. After BRTO, he received C-ion RT as a radical treatment for HCC. After treatment, HCC was well controlled; however, at 35 months post-initiation of C-ion RT, he developed local recurrence without a further reduction in liver function status. Therefore, we repeated C-ion RT. The patient remains alive at 3 months post-treatment, with no evidence of local recurrence, distant metastasis, or toxicity. Although this is a single case report, it suggests that a combinatorial treatment consisting of BRTO and C-ion RT may increase survival rates of patients with HCC and HE.
\end{abstract}

Keywords Hepatocellular carcinoma $\cdot$ Hepatic encephalopathy $\cdot$ Carbon-ion radiotherapy $\cdot$ Balloon-occluded retrograde transvenous obliteration

\section{Introduction}

Liver function is one of the most important prognostic factors with respect to treatment of hepatocellular carcinoma (HCC); indeed, patients with poor liver function are more difficult to cure [1,2]. Although hepatic resection is the first choice treatment for HCC, patients with poor liver function (i.e., the Barcelona Clinic Liver Cancer [BCLC] classification stage B and C), and/or hepatic encephalopathy (HE), and/or portal hypertension are contraindicated $[1,2]$. Percutaneous radiofrequency ablation (RFA) and transarterial chemoembolization (TACE) are viable treatment options for

Kei Shibuya

1 Department of Radiation Oncology, Gunma University Graduate School of Medicine, 3-39-22, Showa-machi, Maebashi, Gunma 371-8511, Japan

2 Gunma University Heavy Ion Medical Center, 3-39-22, Showa-machi, Maebashi, Gunma 371-8511, Japan inoperable patients (i.e., those with BCLC stage 0, A, B, and those that refuse surgery). However, these treatments have limitations. It is difficult to apply RFA to large tumors, lesions adjacent to the large vessels, lesions that are undetectable by ultrasound, and in cases where portal blood flow is reduced by a portosystemic shunt; such patients are at high risk of liver-related complications and exacerbation of $\mathrm{HE}$ after TACE.

Use of carbon-ion radiotherapy (C-ion RT) to treat HCC has shown favorable clinical results, with minimal liver damage [3-6]. These favorable results are due to the biological and physical advantages that C-ion RT has over X-ray RT [7-9]. However, C-ion RT for HCC with HE presents technical difficulties in patients who cannot maintain a static position during irradiation due to flapping tremor or disturbance of consciousness. Therefore, it is necessary to control $\mathrm{HE}$ before C-ion RT. In this respect, balloon-occluded retrograde transvenous obliteration (BRTO) is considered an effective treatment. 
Here, we report a patient with HCC with a favorable clinical course who received sequential treatment with BRTO and C-ion RT. For this patient, standard therapies were not considered applicable.

\section{Case report}

A 78-year-old Japanese male with HCC was referred to the Gunma University Heavy Ion Medical Center for C-ion RT. He had liver cirrhosis caused by infection with hepatitis $\mathrm{C}$ virus (genotype $2 \mathrm{a}$ ), which was previously treated with interferon/ribavirin, but a sustained virologic response was not achieved. His performance status was 1 . Liver function status was Child-Pugh class C (score: 10), with grade III HE (evaluated according to West Haven criteria), BCLC stage $\mathrm{C}$, and modified albumin-bilirubin (mALBI) grade 3 (score: $-1.15)[2,10]$. Laboratory tests showed elevated levels of serum ammonia $(241 \mu \mathrm{g} / \mathrm{dL}$; institution reference range 3-47 $\mu \mathrm{g} / \mathrm{dL}$ ), alpha-fetoprotein (3.5 IU/L; reference range $0-7.0 \mathrm{IU} / \mathrm{L}$ ), and des- $\gamma$-carboxy prothrombin (DCP) (83 AU/ $\mathrm{mL}$; reference range $<40 \mathrm{AU} / \mathrm{mL})$, total bilirubin $(1.3 \mathrm{mg} /$ $\mathrm{dL}$; reference range $0.3-1.2 \mathrm{mg} / \mathrm{dL})$, albumin $(2.4 \mathrm{mg} / \mathrm{dL}$; reference range $3.9-5.0 \mathrm{mg} / \mathrm{dL}$ ), prothrombin time $(53 \%$; reference range $70-130 \%)$, platelets $(78,000$ cells $/ \mu \mathrm{L}$; reference range 160,000-350,000 cells/ $\mu \mathrm{L}), \mathrm{HCV}-\mathrm{RNA}(5.2$ $\log _{10} \mathrm{IU} / \mathrm{mL}$ ). He was also anti-HCV antibody positive. The indocyanine green retention rate at $15 \mathrm{~min}$ (ICG15) was $51.4 \%$. Previously, HE was treated with systemic medication (rifaximin) and branched chain amino acids. Computed tomography (CT) and gadolinium-ethoxybenzyl-diethylenetriamine pentaacetic acid (Gd-EOB-DTPA)-enhanced magnetic resonance imaging (MRI) revealed a nodular tumor $(2.6 \times 2.5 \times 2.1 \mathrm{~cm})$ in segment eight, with early enhancement in the arterial phase and slight washout in the delayed phase (Fig. 1a, b). MRI and CT showed a portosystemic shunt from the splenic vein to the left renal vein (splenorenal shunt) (Fig. 2), no ascites, and no evidence of metastasis to lymph nodes and distant organs. The patient was diagnosed with Stage IB (clinical T1bNOM0) HCC according to the 8th edition of the Union for International Cancer Control/ American Joint Committee on Cancer TNM staging system.

Surgery, RFA, and TACE were not indicated due to poor liver function (mALBI grade $2 b$; ICG15, 51.4\%), tumor location (adjacent to vascular structures), and portal hypertension with a portosystemic shunt. Therefore, C-ion RT was considered as an alternative treatment option for $\mathrm{HCC}$, however, it was deemed difficult due to impaired consciousness caused by exacerbation of HE. The splenorenal shunt was considered to be the main cause of HE; therefore, the treatment strategy was discussed with the cancer board of our hospital, and C-ion RT was recommended after HE was improved by occluding the splenorenal shunt with BRTO (Fig. 3).

After BRTO, HE, serum ammonia levels, and liver function improved immediately with no exacerbation of ascites (Table 1; Fig. 4). Despite the improvement in liver function, there was no change in the treatment strategy for HCC because poor liver function (mALBI grade, 2b; ICG15, $44.3 \%$ ) precluded surgery and C-ion RT potentially has a higher local effect than TACE [7]. C-ion RT began 1 month after BRTO. The dose of C-ion RT was 60.0 Gy [Relative Biological Effectiveness (RBE)], delivered in four fractions (Fig. 1c). The patient completed C-ion RT with no acute toxicity. After C-ion RT, there was no evidence of local recurrence, distant metastasis, or toxicity for about 3 years (i.e., no recurrence of HE, no obvious exacerbation of liver function, and no acute exacerbation of HCV infection) (Table 2; Fig. 1d). After the tumor disappeared following C-ion RT, he was treated with a 12-week course of glecaprevir/pibrentasvir (GLE/PIB), which resulted in a sustained virologic response (SVR).

However, at 35 months from initiation of C-ion RT, MRI revealed local recurrence for HCC, with no evidence of metastasis to lymph nodes or distant organs (Fig. 5a, b). Liver function status at the time of local recurrence was Child-Pugh class A (score: 5), BCLC stage C, and mALBI grade $2 \mathrm{~b}$ (score: -2.25 ). The ICG15 was $41.6(\%)$. Therefore, we scheduled and performed re-irradiation with $\mathrm{C}$-ion $\mathrm{RT}$ at $60.0 \mathrm{~Gy}$ [RBE], delivered in four fractions (Fig. 5c). The patient completed C-ion RT without any acute toxicity. Three months after C-ion RT, CT showed tumor shrinking (Fig. 5d) and there was no evidence of local recurrence, distant metastasis, or recurrence of HE.

\section{Discussion}

Here, we show that BRTO can control HE and enable radical treatment for $\mathrm{HCC}$ with $\mathrm{C}$-ion RT. In addition, there was no exacerbation of poor liver function, meaning that $\mathrm{C}$-ion $\mathrm{RT}$ could be repeated to treat the recurrent lesion. BRTO and $\mathrm{C}$-ion RT for HCC may prolong survival in this case.

Retrograde transvenous obliteration (RTO), including BRTO, is an established treatment for gastric varices [11, 12]. However, RTO is not an established treatment for HE, although the efficacy and safety of RTO as a treatment for HE have been reported [12-14]. Lee et al. reported 91\% clinical cases of HE that were treated successfully by RTO; follow-up was over 2 years, with low rates of tolerable toxicity [14]. In addition, $67 \%$ of patients had complete resolution of their HE symptoms during a follow-up period of $893 \pm 585$ days (range 36-1881 days, median 755.0 days). Ishikawa et al. reported that BRTO significantly improves hyperammonemia and liver function in portal hypertensive 
Fig. 1 Contrast-enhanced computed tomography (CT) and dose distribution of carbonion radiotherapy (C-ion $\mathrm{RT}$ ) for hepatocellular carcinoma (HCC). a Axial CT image taken in the arterial phase before C-ion RT. The white arrow shows $\mathrm{HCC}$ with arterial enhancement. b CT image taken in the delayed phase. The white arrow shows slight washout. c Dose distribution of C-ion RT. The area within the red outline is the gross tumor volume (GTV). Highlighted are the $95 \%$ (red), $90 \%$ (orange), $80 \%$ (yellow), 65\% (green), $50 \%$ (blue), and $20 \%$ (purple) isodose curves $(100 \%=60.0 \mathrm{~Gy}$ [RBE]). d CT image taken in the arterial phase 6 months after primary C-ion RT, showing disappearance of the tumor

Fig. 2 Computed tomography (CT) images showing the splenorenal shunt. a Axial CT image. b Coronal CT image. The white arrow shows the splenorenal shunt
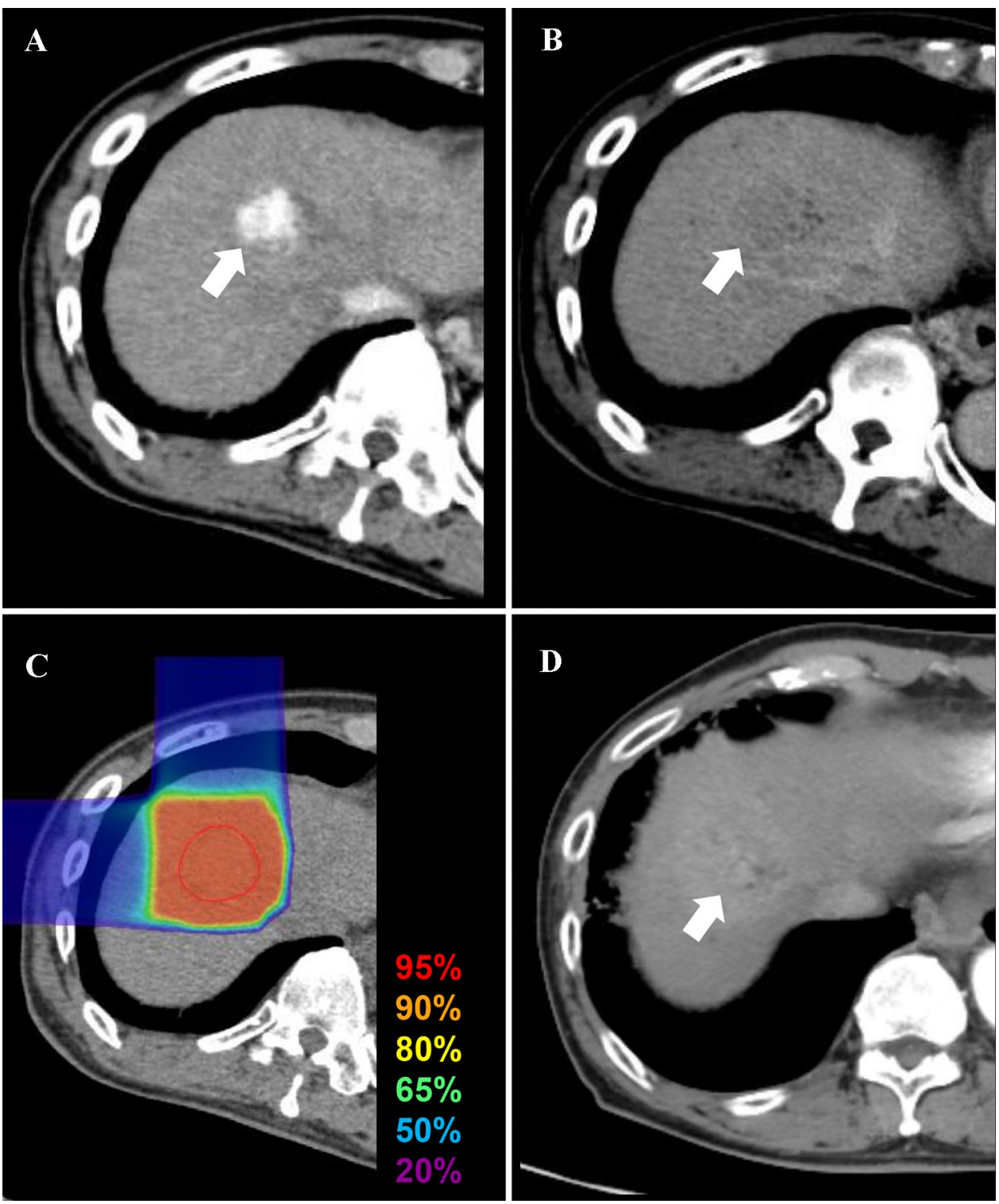
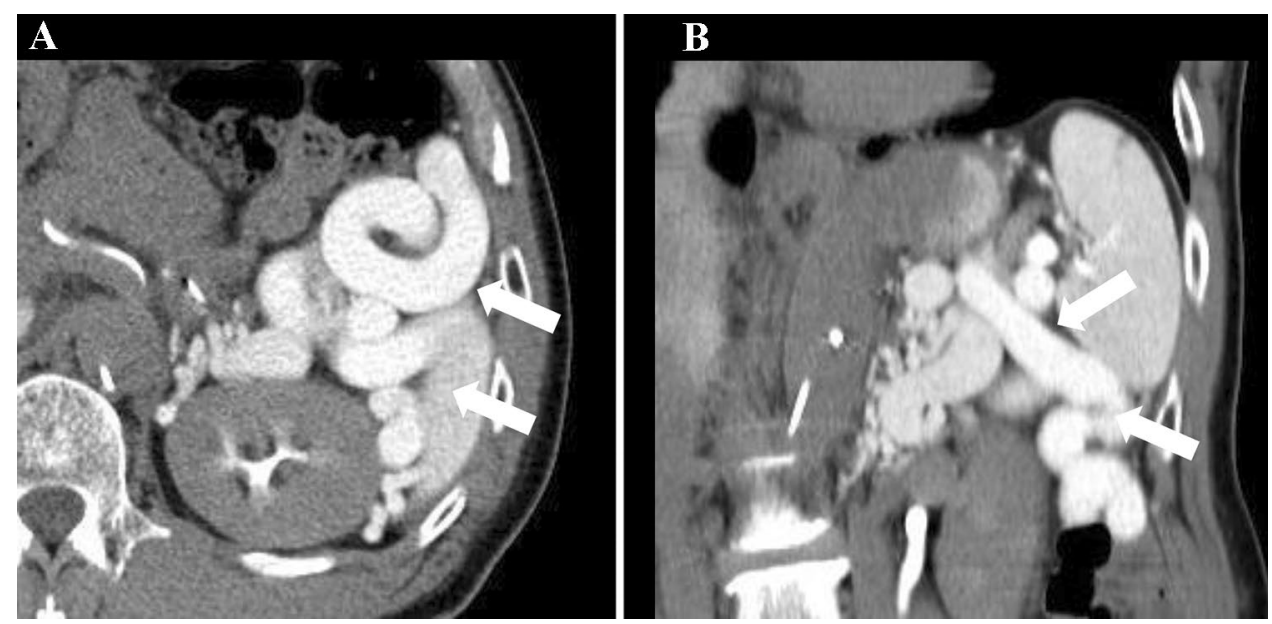
Fig. 3 Angiography before and after balloon-occluded retrograde transvenous obliteration (BRTO). a Before BRTO. b After BRTO, showing disappearance of the shunt flow. The white arrow shows the splenorenal shunt

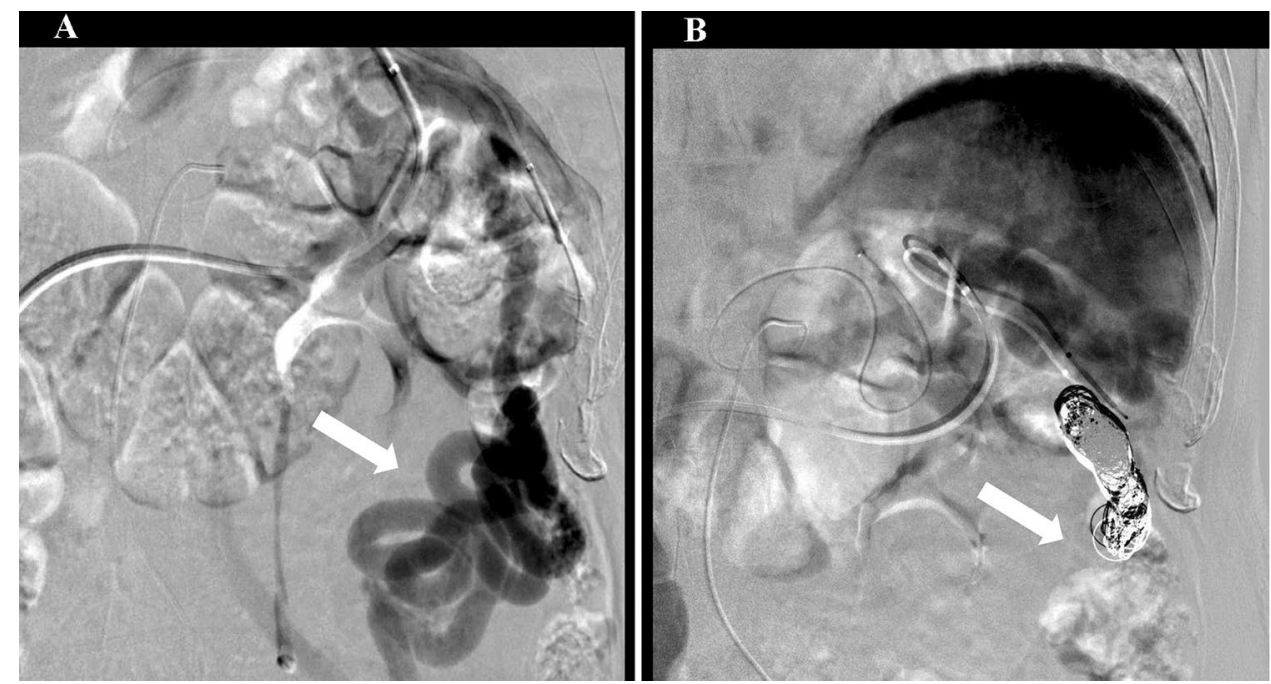

Table 1 Parameters of liver function before and after BRTO

\begin{tabular}{lll}
\hline & Before BRTO & 1 month after BRTO \\
\hline HE & Grade III & Grade 0 \\
Ascites & None & None \\
T-bil (mg/dL) & 1.3 & 1.3 \\
Alb (mg/dL) & 2.4 & 2.8 \\
PT ratio (\%) & 53 & 77 \\
ICG15 (\%) & 51.4 & 44.3 \\
Child-Pugh & C (score: 10$)$ & A (score: 6$)$ \\
mALBI grade & 3 (score: -1.15$)$ & 2b (score: -1.49$)$ \\
\hline
\end{tabular}

$H E$ hepatic encephalopathy (evaluated by West Haven criteria), T-bil total bilirubin (reference range 0.3-1.2), Alb albumin (reference range 3.9-5.0), $P T$ prothrombin time (reference range 70-130), ICG15 indocyanine green retention rate at $15 \mathrm{~min}, B R T O$ balloon-occluded retrograde transvenous obliteration

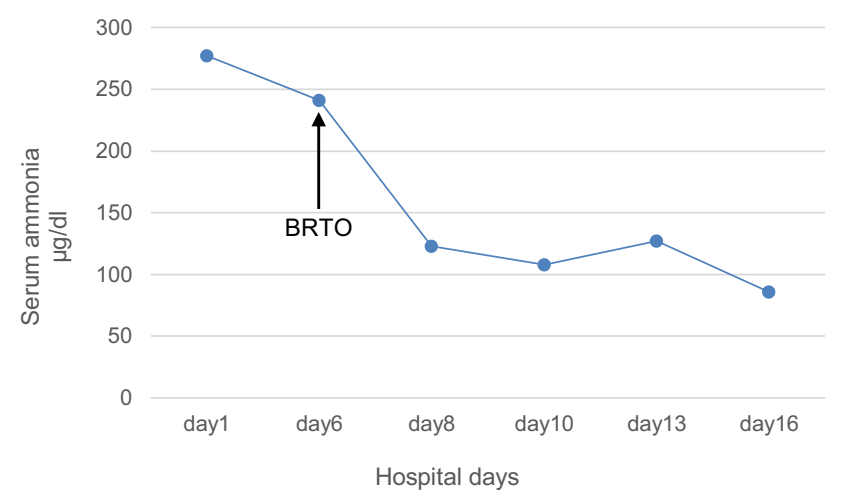

Fig. 4 Kinetics of serum ammonia levels before and after balloonoccluded retrograde transvenous obliteration. BRTO balloon-occluded retrograde transvenous obliteration patients with refractory HE by increasing portal flow [15]. In fact, there was an improvement in HE and liver function after BRTO in our case. To the best of our knowledge, no report has described use of BRTO for HCC patients with HE prior to radical treatment. Our results show that BRTO is an option that may enable HCC patients with HE to receive radical local treatment.

C-ion RT delivers higher local doses than X-ray RT (i.e., stereotactic body RT and intensity-modulated RT) [8, 9]; also, the modality enables delivery of a reduced dose to the liver while ensuring good target coverage. Therefore, the effect on liver function is minimal, and exacerbation of poor liver function and radiation-induced liver disease after $\mathrm{C}$-ion RT are rare [3-6]. This ability to preserve liver function might contribute to survival [16]. In this case, there was no obvious exacerbation of the Child-Pugh class or mALBI grade after $\mathrm{C}$-ion $\mathrm{RT}$, and preservation of liver function enabled re-irradiation with $\mathrm{C}$-ion $\mathrm{RT}$ to treat local recurrence. We believe that radical cancer treatment with $\mathrm{C}$-ion RT, coupled with preservation of liver function, prolonged survival in this case.

\section{Conclusion}

This report suggests that a combinatorial treatment consisting of BRTO and C-ion RT may prolong survival of patients with HCC and HE. This is because combination treatment enables HCC patients with HE to receive radical local treatment. 
Table 2 Laboratory test data before and after C-ion RT

\begin{tabular}{|c|c|c|c|c|c|c|}
\hline & Before C-ion RT & 3 months & 6 months & 12 months & 15 months & 27 months \\
\hline $\mathrm{HE}$ & Grade 0 & Grade 0 & Grade 0 & Grade 0 & Grade 0 & Grade 0 \\
\hline Ascites & None & None & None & None & None & None \\
\hline T-bil (mg/dL) & 1.3 & 1.4 & 2.0 & 2.7 & 1.7 & 1.9 \\
\hline Alb (mg/dL) & 3.0 & 3.1 & 3.2 & 2.9 & 3.1 & 3.5 \\
\hline PT ratio $(\%)$ & 76 & 75 & 75 & 74 & 76 & 79 \\
\hline Child-Pugh & A (score: 6) & A (score: 6) & B (score: 7) & B (score: 7) & A (score: 6) & A (score: 6) \\
\hline mALBI grade & 2b (score: - 1.66) & $2 \mathrm{~b}$ (score: -1.72 ) & $2 \mathrm{~b}$ (score: -1.71$)$ & 3 (score: -1.37 ) & 2b (score: -1.67 ) & $2 \mathrm{~b}$ (score: -1.98$)$ \\
\hline HCV-RNA $\left(\log _{10} \mathrm{IU} / \mathrm{mL}\right)$ & 5.2 & 5.8 & 6.1 & N.D & N.D & N/A \\
\hline
\end{tabular}

C-ion RT carbon-ion radiotherapy, N.D. not detected, N/A not applicable

Fig. 5 Computed tomography (CT), magnetic resonance imaging (MRI), and dose distribution of carbon-ion radiotherapy (C-ion RT) for local recurrence of hepatocellular carcinoma (HCC). a Axial CT image taken in the arterial phase. The white arrow shows recurrence of $\mathrm{HCC}$ with arterial enhancement. b CT image taken in the delayed phase. The white arrow shows slight washout. c Dose distribution of C-ion RT for recurrent HCC. The area within the red outline is the GTV. Highlighted are the $95 \%$ (red), $90 \%$ (orange) $80 \%$ (yellow), 65\% (green), $50 \%$ (blue), and $20 \%$ (purple) isodose curves $(100 \%=60.0 \mathrm{~Gy}$ [RBE]). $\mathbf{d}$ MRI image taken in the arterial phase three months after re-irradiation with C-ion RT. The white arrow shows tumor shrinkage
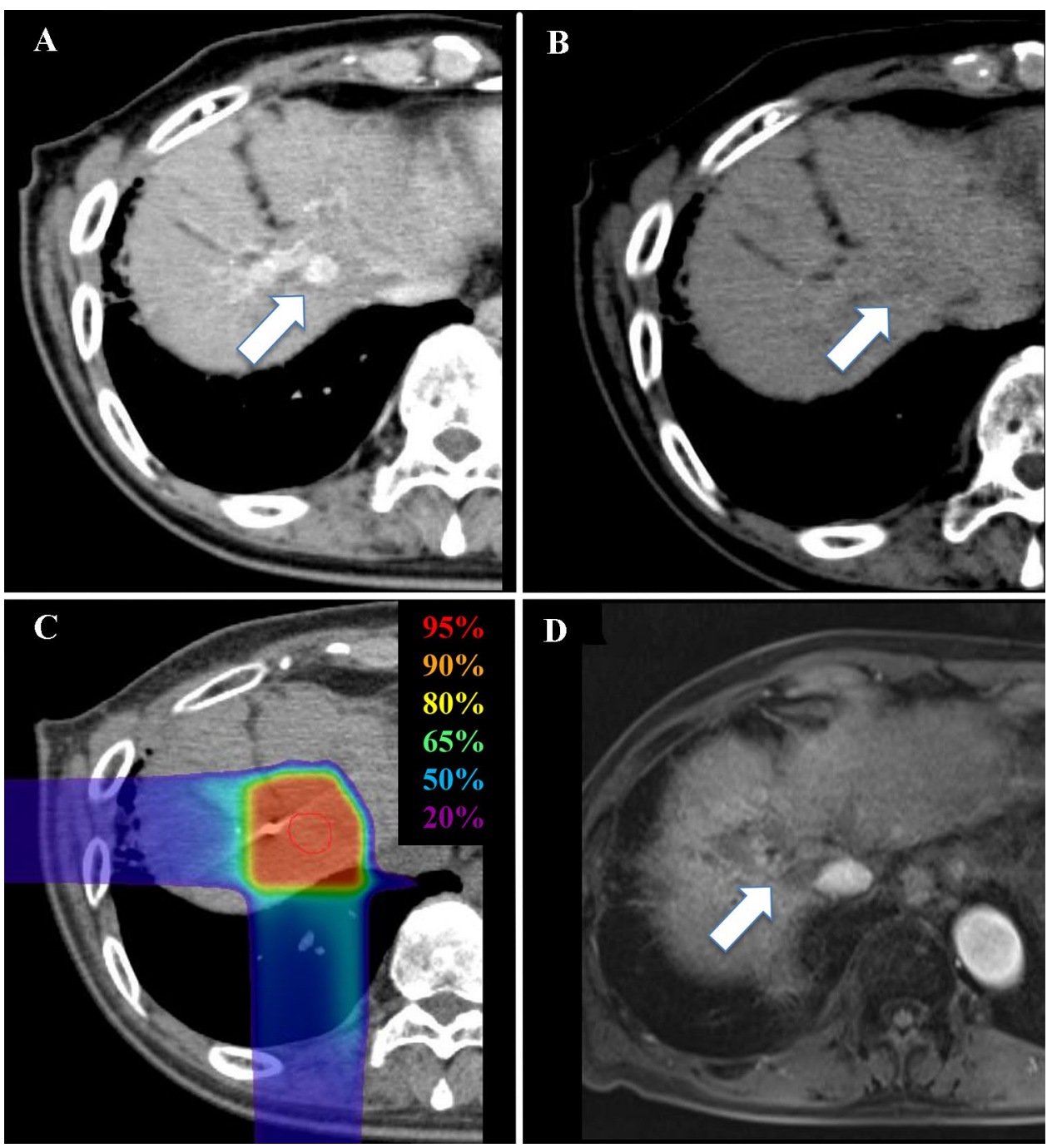
Acknowledgements The authors thank our colleagues at Gunma University Heavy Ion Medical Center, and the Department of Radiation Oncology Gunma University Graduate School of Medicine.

Funding This work was supported by the Grants-in-Aid from the Ministry of Education, Culture, Sports, Science, and Technology of Japan for programs for Leading Graduate Schools, Cultivating Global Leaders in Heavy Ion Therapeutics and Engineering. The funding body played no role in study design, data collection, analysis, and interpretation, or in writing of the manuscript.

\section{Declaration}

Conflict of interest Naoto Osu, Shintaro Shiba, Kei Shibuya, Shohei Okazaki, Yuhei Miyasaka, Masahiko Okamoto and Tatsuya Ohno declare that they have no conflict of interest.

Human/animal rights All procedures followed have been performed in accordance with the ethical standards laid down in the 1964 Declaration of Helsinki and its later amendments.

Informed consent Informed consent was obtained from the patients for being included in the study.

Open Access This article is licensed under a Creative Commons Attribution 4.0 International License, which permits use, sharing, adaptation, distribution and reproduction in any medium or format, as long as you give appropriate credit to the original author(s) and the source, provide a link to the Creative Commons licence, and indicate if changes were made. The images or other third party material in this article are included in the article's Creative Commons licence, unless indicated otherwise in a credit line to the material. If material is not included in the article's Creative Commons licence and your intended use is not permitted by statutory regulation or exceeds the permitted use, you will need to obtain permission directly from the copyright holder. To view a copy of this licence, visit http://creativecommons.org/licenses/by/4.0/.

\section{References}

1. EASL-EORTC clinical practice guidelines: management of hepatocellular carcinoma. J Hepatol. 2012;56:908-43.

2. Llovet JM, Brú C, Bruix J. Prognosis of hepatocellular carcinoma: the BCLC staging classification. Semin Liver Dis. 1999;19:329-38.

3. Shibuya K, Ohno T, Katoh H, et al. A feasibility study of highdose hypofractionated carbon ion radiation therapy using four fractions for localized hepatocellular carcinoma measuring $3 \mathrm{~cm}$ or larger. Radiother Oncol. 2019;132:230-5.

4. Shiba S, Abe T, Shibuya K, et al. Carbon ion radiotherapy for 80 years or older patients with hepatocellular carcinoma. BMC Cancer. 2017;17:721.
5. Kasuya G, Kato H, Yasuda S, et al. Progressive hypofractionated carbon-ion radiotherapy for hepatocellular carcinoma: combined analyses of 2 prospective trials. Cancer. 2017;123:3955-65.

6. Shiba S, Shibuya K, Okamoto M, et al. Clinical impact of hypofractionated carbon ion radiotherapy on locally advanced hepatocellular carcinoma. Radiat Oncol. 2020;15:195.

7. Shiba S, Shibuya K, Katoh H, et al. A comparison of carbon ion radiotherapy and transarterial chemoembolization treatment outcomes for single hepatocellular carcinoma: a propensity score matching study. Radiat Oncol. 2019;14:137.

8. Abe T, Saitoh J, Kobayashi D, et al. Dosimetric comparison of carbon ion radiotherapy and stereotactic body radiotherapy with photon beams for the treatment of hepatocellular carcinoma. Radiat Oncol. 2015;10:187.

9. Shiba S, Shibuya K, Kawashima M, et al. Comparison of dose distributions when using carbon ion radiotherapy versus intensitymodulated radiotherapy for hepatocellular carcinoma with macroscopic vascular invasion: a retrospective analysis. Anticancer Res. 2020;40:459-64.

10. Johnson PJ, Berhane S, Kagebayashi C, et al. Assessment of liver function in patients with hepatocellular carcinoma: a new evidence-based approach-the ALBI grade. J Clin Oncol. 2015;33:550-8.

11. Fukuda T, Hirota S, Sugimura K. Long-term results of balloonoccluded retrograde transvenous obliteration for the treatment of gastric varices and hepatic encephalopathy. J Vasc Interv Radiol. 2001;12:327-36.

12. Lee EW, Lee AE, Saab S, et al. Retrograde transvenous obliteration (RTO): a new treatment option for hepatic encephalopathy. Dig Dis Sci. 2020;65:2483-91.

13. Laleman W, Simon-Talero M, Maleux G, et al. Embolization of large spontaneous portosystemic shunts for refractory hepatic encephalopathy: a multicenter survey on safety and efficacy. Hepatology. 2013;57:2448-57.

14. Lee EW, Saab S, Kaldas F, et al. Coil-assisted retrograde transvenous obliteration (CARTO): an alternative treatment option for refractory hepatic encephalopathy. Am J Gastroenterol. 2018;113:1187-96.

15. Ishikawa T, Sasaki R, Nishimura T, et al. Comparison of patients with hepatic encephalopathy and those with gastric varices before and after balloon-occluded retrograde transvenous obliteration. Hepatol Res. 2018;48:1020-30.

16. Mathurin P, Rixe O, Carbonell N, et al. Review article: overview of medical treatments in unresectable hepatocellular carcinoma-an impossible meta-analysis? Aliment Pharmacol Ther. 1998;12:111-26.

Publisher's Note Springer Nature remains neutral with regard to jurisdictional claims in published maps and institutional affiliations. 